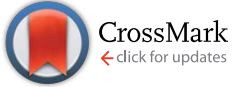

Cite this: RSC Adv., 2017, 7, 13827

Received 15th January 2017

Accepted 23rd February 2017

DOI: $10.1039 / c 7 r a 00636 e$

rsc.li/rsc-advances

\section{On-surface manipulation of atom substitution between cobalt phthalocyanine and the $\mathrm{Cu}(111)$ substrate $\uparrow$}

\author{
Kongchao Shen,,$^{\mathrm{ab}}$ Bai Narsu, $\mathrm{f}^{\mathrm{c}}$ Gengwu Ji, Haoliang Sun, ${ }^{\mathrm{b}} \mathrm{Jinbang} \mathrm{Hu},{ }^{\mathrm{b}}$ \\ Zhaofeng Liang, ${ }^{b}$ Xingyu Gao, ${ }^{b}$ Haiyang Li, ${ }^{a}$ Zheshen Li, ${ }^{d}$ Bo Song, ${ }^{\text {*e }}$ Zheng Jiang, $^{\text {*b }}$ \\ Han Huang, ${ }^{f}$ Justin W. Wells ${ }^{g}$ and Fei Song ${ }^{\star b}$
}

On-surface fabrication of controllable nanostructures is an appealing topic in the field of molecular electronics. Herein, the adsorption of cobalt phthalocyanine (CoPc) on a $\mathrm{Cu}(111)$ surface is investigated utilizing a combination of photoelectron spectroscopy (PES) and density functional theory (DFT). Interestingly, the scenario of atom exchange is discovered at the interface at room temperature (RT), namely the substitution of the cobalt atom in $\mathrm{CoPc}$ by a surface $\mathrm{Cu}$ adatom. Moreover, thermal annealing enhances the substitution process considerably which is demonstrated to be complete at about $573 \mathrm{~K}$. As revealed by DFT calculations, the driving force for the observed interface transmetalation is most probably provided by the initial strong molecular-substrate interaction between Co atoms and the $\mathrm{Cu}(111)$ surface, the external thermodynamic energy gained from thermal sublimation and thermal annealing, and the tendency to form Co-Cu alloy at the interface. While CoPc has been successfully utilized in electrocatalysts for fuel cell applications and CuPc is commonly used as a leading material in organic solar cells, this report of interface transmetalation from CoPc to CuPc in a solid state environment may offer an encouraging approach towards the artificial engineering of organometallic nanostructures and related properties for surface catalysts, molecular electronics and so on.

\section{Introduction}

Controllable manipulation of organic nanostructures has been widely utilized as a promising approach for tuning the electronic, optical, chemical or physical properties at the surface/ interface, with appealing application manifested in molecular electronics and biosensors. ${ }^{\mathbf{1}}$ Fabrication of artificial on-surface structures is therefore a key procedure, including both the bottom-up method (for example, molecular self-assembly) and

\footnotetext{
${ }^{a}$ Department of Physics, Zhejiang University, Hangzhou, 310027, China ${ }^{b}$ Shanghai Synchrotron Radiation Facility, Shanghai Institute of Applied Physics, Chinese Academy of Sciences, Shanghai, 201204, China. E-mail: jiangzheng@sinap. ac.cn; songfei@sinap.ac.cn

'Inner Mongolia Key Lab for Physics and Chemistry of Functional Materials, Department of Physics, Inner Mongolia Normal University, Hohhot, 010022, China

${ }^{d}$ ISA, Department of Physics and Astronomy, University of Aarhus, 8000 Aarhus C, Denmark

${ }^{e}$ University of Shanghai for Science and Technology, Shanghai, 200093, China. E-mail: bsong@usst.edu.cn

${ }^{f}$ School of Physics and Electronics, Central South University, Changsha, 410083, China ${ }^{g}$ Department of Physics, Norwegian University of Science and Technology, Trondheim N7491, Norway

† Electronic supplementary information (ESI) available. See DOI: 10.1039/c7ra00636e

\$ These authors contributed equally to this work.
}

the top-down technique. The family of transition metal phthalocyanines (TMPcs, a $\pi$ electron conjugated ring system complex) has been for a long period the subject of intense interest, due to their unique electronic and optical properties in biological processes, ${ }^{2,3}$ catalysis, ${ }^{4-8}$ field-effect transistors, ${ }^{9}$ solar cells ${ }^{10}$ and the dye industry. ${ }^{11}$ As realized, the performance of TMPc molecules in devices can be easily affected by the interface interactions. ${ }^{\mathbf{1 2 - 1 5}}$ For example, the contact to specific substrate can alter or suppress the electronic properties of the TMPcs. Consequently, detailed investigations at molecular level of the interface between TMPcs and metallic substrates is necessary and will continually shed valuable insight into the understanding of various processes taking place at device interfaces.

Due to the presence of partially empty d orbitals of the central metal atom in TMPcs, molecular substrate interaction can be systematically tuned depending on its contribution to the formation of interface structures. Therefore, well control on the transitional metal center incorporated into Pc macrocycles is of utmost importance to achieve tailored properties. For instance, the interface electronic structure has been studied for FePc, ZnPc, CuPc et al. on metallic substrates, and it was found that depending on the transition metal, the d-levels of metal atoms can significantly contribute to the highest occupied molecular orbital (HOMO) or lowest unoccupied molecular 
orbital (LUMO) of the TMPc. ${ }^{16-18}$ In particular, CoPc is a paramagnetic molecule with the cobalt ion interacts with its surrounding giving rise to different electronic and magnetic configurations, ${ }^{19}$ and is therefore a prototype candidate in view of a possible implementation of molecular magnetic junctions and electrocatalysts. ${ }^{20}$ Moreover, it has been repeatedly demonstrated in the literature that the cobalt atom in CoPc interacts strongly with metal substrates $(\mathrm{Au}, \mathrm{Cu}$ or $\mathrm{Ag}))^{15,20-23}$ which might lead to the structural modification of the CoPc adsorbate, for example, the incorporation of surface adatom into the Pc macrocycle from the substrate and finally the substitution of central Co atom. This atom exchange process is the extension of on-surface metalation scenario, and usually called transmetalation in the form of $\mathrm{M}_{1} \mathrm{Pc}+\mathrm{M}_{2} \rightarrow \mathrm{M}_{2} \mathrm{Pc}+\mathrm{M}_{1}$ which has been demonstrated in solution before. ${ }^{24,25}$ As reported by Doyle et al. for nickel porphyrin compounds (NiDBrDPP and NiTBrPP) adsorbed on a $\mathrm{Cu}(111)$ surface, transmetalation at interface was discovered upon the contact with metal support and was enhanced by thermal annealing where nickel was completely removed from the porphyrin core and replaced by a substrate copper atom. ${ }^{26}$ However, on-surface transmetalation of transitional phthalocyanine macrocycle has not been observed before in solid-state conditions.

Herein we investigate the growth of CoPc on a $\mathrm{Cu}(111)$ surface with particular attention to explore the variation of interface structures and the related electronic properties. Surprisingly, it is shown in our study that CoPc interacts strongly with the $\mathrm{Cu}(111)$ substrate leading to the substitution of the $\mathrm{Co}$ ion by a $\mathrm{Cu}$ adatom from the substrate with the assistance of external thermodynamic energy, which subsequently results in the formation of Cu-metallated phthalocyanine and the releasing of metal cobalt atoms at interface.

\section{Results and discussion}

At the organic film/metal interfaces, various scenarios can be found: formation of the dipole layer due to charge transfer, redistribution of electron cloud, and interfacial chemical reaction, ${ }^{27}$ where the chemical interactions may result in new features/states at interface. As well known, XPS is a very powerful tool at detecting the changes in chemical state and environment of an atom, and will therefore provide fingerprint information regarding on the process occurring at $\mathrm{CoPc} /$ $\mathrm{Cu}(111)$ interface. First, $1 \mathrm{ML}$ CoPc was prepared on the $\mathrm{Cu}(111)$ surface under UHV at RT followed by thermal annealing to $373 \mathrm{~K}, 473 \mathrm{~K}, 573 \mathrm{~K}, 673 \mathrm{~K}, 723 \mathrm{~K}$ for 30 minutes, respectively, while the corresponding core levels of $\mathrm{Cu} 2 \mathrm{p}_{3 / 2}$, Co $2 \mathrm{p}_{3 / 2}, \mathrm{~N} 1 \mathrm{~s}$, and $\mathrm{C} 1 \mathrm{~s}$ were recorded after each annealing treatment. Similarly, thick film of CoPc (around $3 \mathrm{ML}$ ) was also evaporated onto the $\mathrm{Cu}$ substrate afterwards and treated with the above-mentioned step-by-step annealing for direct comparison.

To explore possible interactions at the interface involving the central metal atom of CoPc, Co $2 \mathrm{p}$ core level photoemission spectra was investigated in detail. In Fig. 1, the evolution of the Co $2 \mathrm{p}_{3 / 2}$ core level at the $\mathrm{CoPc} / \mathrm{Cu}(111)$ interface are depicted as a function of annealing temperature for the thickness of $1 \mathrm{ML}$
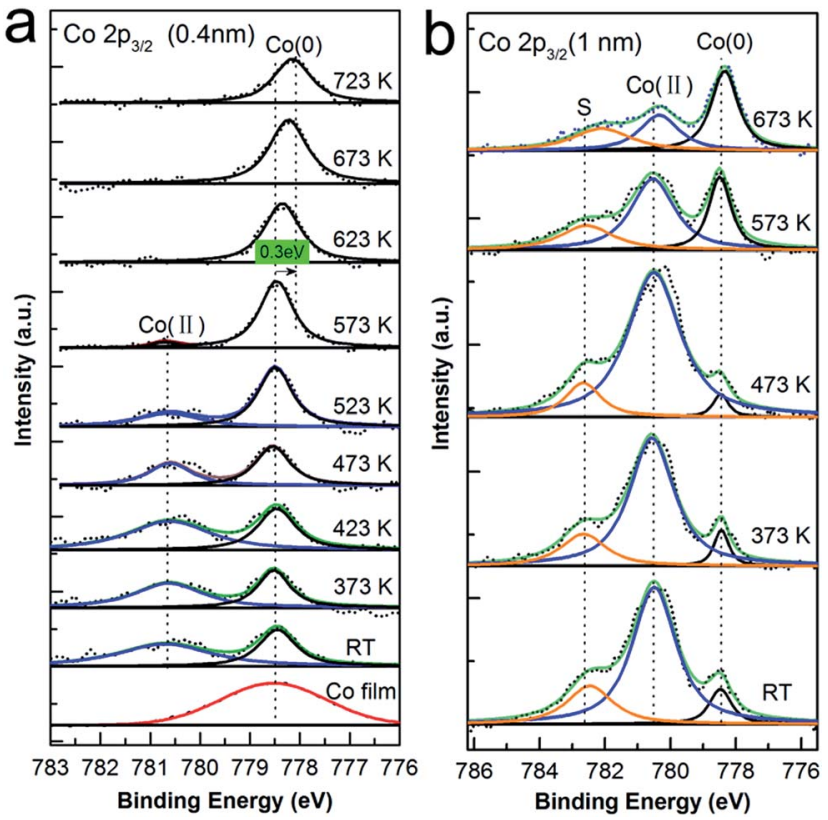

Fig. 1 Co $2 p_{3 / 2}$ XP spectra for the deposition of CoPc on $\mathrm{Cu}(111)$ kept at RT followed by subsequent annealing. (a) Co $2 p_{3 / 2}$ core level of $1 \mathrm{ML}$ and (b) 3 ML CoPc deposited on the Cu surface. Black dots: raw data, blue line: fitted component from Co(॥) oxidation state, black line: fitted $\mathrm{Co}(0)$ component, orange line: the shake-up component related with the Co(॥) state. As a reference, thin Co metal film (around $4 \AA$ ) was also prepared on the $\mathrm{Cu}(111)$ surface as shown in the bottom of (a) with red curve.

and $3 \mathrm{ML} \mathrm{CoPc}$, respectively. At the first glance, a significant change of peak shape is observed by increasing annealing temperature. As seen, the second bottom spectrum in Fig. 1(a) for the as-deposited CoPc layer at RT is dominated by two components: a broad feature at high binding energy at $780.6 \mathrm{eV}$ assigned to the $\mathrm{Co}(\mathrm{II})$ oxidation state, and an intense peak at lower binding energy of $778.5 \mathrm{eV}$, which can be assigned to the cobalt metal state $(\mathrm{Co}(0)) .{ }^{28}$ For convincing arguing, the spectrum of Co $2 \mathrm{p}_{3 / 2}$ from a Co metal film (around $4 \AA$ ) adsorbed on $\mathrm{Cu}(111)$ was also present at the bottom of Fig. 1 (a) (red curve) as a reference, which matches well with the claimed cobalt $(0)$ state from the $\mathrm{CoPc} / \mathrm{Cu}(111)$ interface. Upon increasing annealing temperature, the $\mathrm{Co}(\mathrm{II})$ component attenuates in intensity and gets more and more faint, with a corresponding increase in the intensity of the $\mathrm{Co}(0)$ component from RT until to $573 \mathrm{~K}$ where the Co(II) component almost vanishes. Furthermore, no obvious change was observed afterwards when further annealing from $573 \mathrm{~K}$ to $723 \mathrm{~K}$, except that the peak originated from $\mathrm{Co}(0)$ state turns broader which might be related to the desorption upon high temperature annealing, and shifts down to lower binding energy by about $0.3 \mathrm{eV}$. Similarly, in the case of $3 \mathrm{ML}$ CoPc adsorbed on the $\mathrm{Cu}(111)$ substrate, $\mathrm{Co}(0)$ component was initially discovered at RT even though the signal of $\mathrm{Co}(\mathrm{II})$ state from multilayer is mostly dominating. Moreover, an additional component at $782.6 \mathrm{eV}$ was resolved in Fig. 1(b), which can be assigned to the shake-up peak associated with $\mathrm{Co}(\mathrm{II})$ oxidation state at thick coverage, ${ }^{23}$ while in the case of monolayer thickness such satellite peak was not visible mostly due to the 
molecular-substrate interaction. Further annealing to $373 \mathrm{~K}$ and $473 \mathrm{~K}$ did not give any difference to the peak shape or relative intensity of $\mathrm{Co}(\mathrm{II})$ and $\mathrm{Co}(0)$ state, however, considerable enhancement of the peak intensity of $\mathrm{Co}(0)$ component was recorded when the annealing temperature went up to $573 \mathrm{~K}$ for multilayer of $\mathrm{CoPc}$ on $\mathrm{Cu}(111)$. Surprisingly, the peak contributed from the $\mathrm{Co}(\mathrm{II})$ oxidation state got much weaker and became equally intense to the peak related to the $\operatorname{Co}(0)$ state at $573 \mathrm{~K}$, and shrunk further to about one third of the $\mathrm{Co}(0)$ component after annealed to $673 \mathrm{~K}$, while the $\mathrm{Co}(0)$ peak was kept almost constant from $573 \mathrm{~K}$ to $673 \mathrm{~K}$. Meanwhile, the satellite peak associated with the $\mathrm{Co}$ (II) component was also reduced in a similar manner.

For convenient comparison, spectrum of monolayer and multilayer CoPc adsorbed on the $\mathrm{Cu}(111)$ surface at RT is also stacked together as shown in Fig. S1 in ESI. $\dagger$ In the case of multilayer, relatively intense feature of $\mathrm{Co}(\mathrm{II})$ state was discovered as compared to the $\mathrm{Co}(0)$ state upon deposition at RT, since additional layers after the first layer are less affected by the $\mathrm{Cu}$ substrate. From the above analysis of Co $2 \mathrm{p}_{3 / 2}$ spectra between the monolayer and multilayer coverage, it is fairly visible that a $\mathrm{Co}(0)$ state is present upon adsorption regardless of the film thickness, which is located at $2.1 \mathrm{eV}$ lower binding energy relative to the pristine $\mathrm{Co}(\mathrm{II})$ state from $\mathrm{CoPc}$, and is almost identical to that of the metallic $\mathrm{Co}$ film on $\mathrm{Cu}(111)$. At this point, the presence of a $\mathrm{Co}(0)$ component in Fig. 1 implicating the appearance of metallic Co atom, might point out that the Co ion was displaced from the phthalocyanine macrocycle by $\mathrm{Cu}$ adatoms from substrate and turned to be metallic atoms at the interface. In addition, such replacement process on the $\mathrm{Cu}(111)$ surface begins at RT, enhanced by thermal annealing which probably provides adequate thermal dynamic energy for the surface $\mathrm{Cu}$ adatom, and finishes at about $573 \mathrm{~K}$ when all the Co ions have already been replaced by $\mathrm{Cu}$ atoms. Moreover, the vanishing of $\mathrm{Co}(\mathrm{II})$ peak at $573 \mathrm{~K}$ at the coverage of $1 \mathrm{ML}$ and the remain of such peak after annealed to $673 \mathrm{~K}$ in the case of multilayer together suggest that the ion exchange process only happens for the first layer contacted with the copper substrate. Notably, the shift of $\mathrm{Co}(0)$ peak to lower binding energy by about $0.3 \mathrm{eV}$ at the monolayer coverage from $573 \mathrm{~K}$ to $623 \mathrm{~K}$ is most probably induced by the polarization screening effect. ${ }^{29,30}$ As known, for atoms in a similar chemical state but adsorbed in the submonolayer, first and second layer, respectively, on a metal surface such shifts between the respective signals are always explained with polarization screening by metallic substrate. $^{31}$

As reported by Chassé et al., charge transfer process involving mainly the Co atom was claimed from the significant changes of Co X-ray absorption spectra at the interface. ${ }^{23}$ Moreover, charge transfer induced strong chemical interaction between CoPc and various metals including silver, gold, and graphene/Ni(111) have also been demonstrated from previous reports, ${ }^{14,21,32-39}$ where it is claimed that such interactions directly affect the initial state of the metal ion and changes the effective oxidation state of the ion itself, resulting in a free Co metallic component shifted by about $2 \mathrm{eV}$ to lower binding energy in the Co $2 \mathrm{p}_{2 / 3}$ spectrum..$^{23,39,40}$ Since the interaction between CoPc and copper is expected to be much stronger compared to silver or gold, and the presence of cobalt metallic state $(\mathrm{Co}(0))$ is undoubtedly evident, it can be concluded that the central atom Co in CoPc macrocycle is displaced by copper adatom from the $\mathrm{Cu}$ substrate, and therefore, atom exchange process-transmetalation is believed to occur at the $\mathrm{CoPc} /$ $\mathrm{Cu}(111)$ interface which forms the new CuPc organic and releasing metallic Co atoms. This procedure happens partially at RT, and gets greatly enhanced by thermal annealing which delivers free $\mathrm{Cu}$ adatoms to the interface from substrate and provides enough dynamic energy for barrier overcoming. However, due to the present of copper bulk substrate, no further information could be drawn from the $\mathrm{Cu} 2 \mathrm{p}$ core level since the spectra are kept unchanged during the whole annealing procedure except the intensity attenuation (as shown in Fig. S2 $\dagger$ ). Similarly, on-surface $\mathrm{Ni}-\mathrm{Cu}$ ion exchange process has also been reported for $\mathrm{Ni}$-porphyrinato on $\mathrm{Cu}(111),{ }^{26}$ where an exchange of the $\mathrm{Ni}$ ion in the macrocycle with a substrate $\mathrm{Cu}$ atom results in $\mathrm{Cu}$ metallated porphyrins and metallic nickel atoms on the surface.

Since the desorption of CoPc molecule was recognized in Fig. 1, one may doubt about the intactness of the macrocycle after high temperature annealing and accordingly the conclusion of $\mathrm{Co}-\mathrm{Cu}$ atom exchange at interface. To avoid such ambiguity, the $\mathrm{N} 1 \mathrm{~s}$ and $\mathrm{C} 1 \mathrm{~s}$ spectra were also recorded during annealing procedure, as shown in Fig. 2 and 3, respectively, meanwhile aiming to exploit the relative role of ligand atoms (C, $\mathrm{N})$ in the interaction with the $\mathrm{Cu}$ substrate and the ion exchange process at the interface. The CoPc molecule has two different
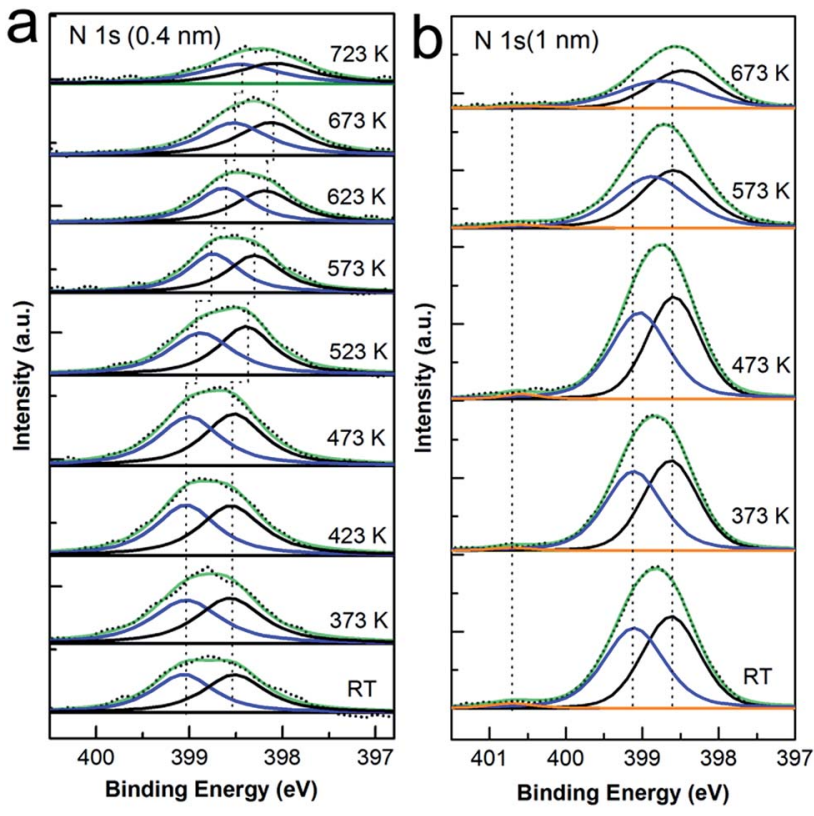

Fig. $2 \mathrm{~N}$ 1s XP spectra of CoPc deposited onto $\mathrm{Cu}(111)$ with different thickness, followed by annealing to the temperatures as indicated. (a) $\mathrm{N}$ 1s core level spectra for $1 \mathrm{ML}$ CoPc and (b) $3 \mathrm{ML}$ CoPc as a function of annealing temperature. Black dots: raw data, black curve: the fitted iminic $\mathrm{N}(-\mathrm{N}=)$ component, blue curve: the fitted metal coordinated $\mathrm{N}$ component, orange curve: the shake-up feature. 

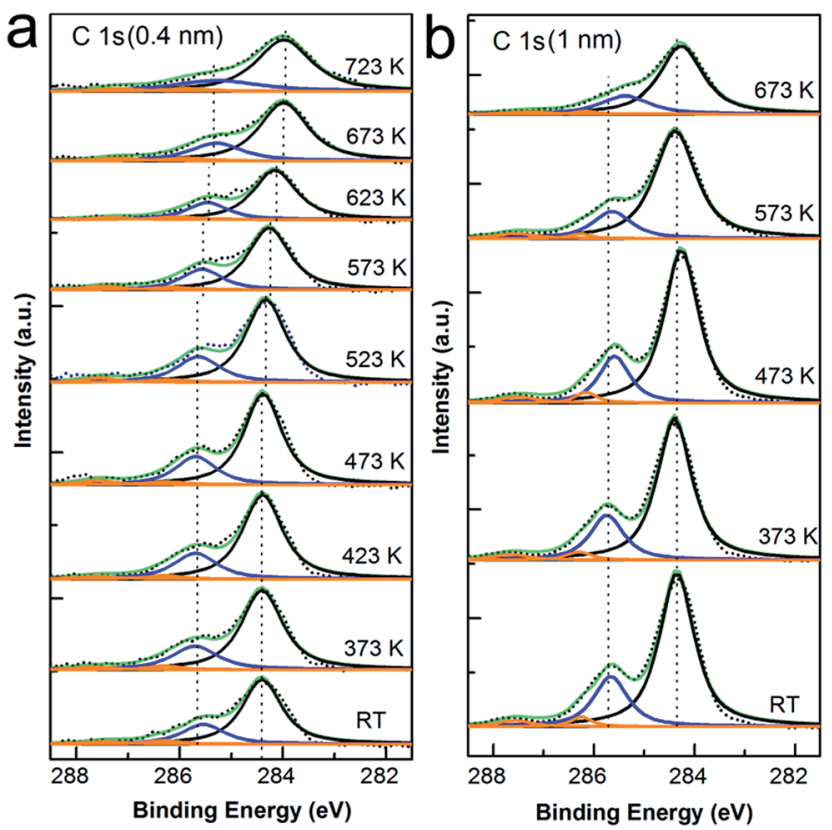

Fig. 3 C 1s XP spectra for varying coverages of CoPc adsorbed on $\mathrm{Cu}(111)$, followed by subsequent annealing, (a) $C$ 1s XP core level spectra for $1 \mathrm{ML}$ and (b) $3 \mathrm{ML}$ CoPc prepared on the Cu substrate. Black dots: raw data, black curve: the component related to $C$ atoms within the benzene ring, blue curve: the component from the pyrrolic $\mathrm{C}$ atoms, orange curves: components from the satellite shake-up.

sites of nitrogen: the iminic nitrogen $(-\mathrm{N}=)$ and the Co bonded nitrogen $(\mathrm{Co}-\mathrm{N})$ in a chemically identical state. ${ }^{\mathbf{4 0}}$ Previous studies show that the metallic nitrogen species is typically located at between 398.3 and $400.6 \mathrm{eV}$ binding energy ${ }^{\mathbf{4 0 - 4 4}}$ and the iminic N in MPc macrocycle is approximately in the binding energy range from 398.4 to $398.9 \mathrm{eV} .^{\mathbf{4 0 , 4 1 , 4 5 , 4 6}}$ In addition, there are 4 nitrogen atoms at iminic site and 4 metal-bonded nitrogen atoms in $\mathrm{CoPc}$, which should give a $1: 1$ ratio of the corresponding peak intensity. As expected, the $\mathrm{N}$ 1s spectra of CoPc monolayer (Fig. 2(a)) and multilayer (Fig. 2(b)) on $\mathrm{Cu}(111)$ both show two well-resolved main peaks (blue and black curve) with approximately equal amplitude, the peak at $398.5 \mathrm{eV}$ can be directly assigned to the iminic nitrogen while the peak at $400.1 \mathrm{eV}$ is related to the Co-bonded nitrogen with the energy difference well consistent with the literature. ${ }^{40}$ Moreover, the evolution of $\mathrm{N}$ 1s peak with increasing annealing temperature is also presented. As seen, the peak shape was kept constant as well as the relative intensity ratio and the binding energy difference of the two components, except that both peak decrease and shift to lower binding energy by about $0.5 \mathrm{eV}$ annealed from $523 \mathrm{~K}$ until to $723 \mathrm{~K}$ in the case of one monolayer CoPc deposition while the shift is almost negligible (less than $0.1 \mathrm{eV}$ ) at multilayer coverage. The attenuation of peak intensity is caused by desorption of CoPc molecules during annealing, which agrees well with the discussion in the Co $2 \mathrm{p}$ core level section. The peak shift can be explained again with the polarization screening effect induced by the metal substrate, which is distinct when the coverage varies from monolayer to submonolayer while ambiguous in the case of multilayer. ${ }^{31,47-52}$
However, there is still one point needed to be considered: the effect of the substitution of Co ion in CoPc by copper atom on the $\mathrm{N}$ 1s core level spectrum. As we pointed out before, the displacement of $\mathrm{Co}$ ion by $\mathrm{Cu}$ adatom results in the formation of CuPc, however, due to relative adjacency in binding energy of Co bonded and $\mathrm{Cu}$ bonded nitrogen ${ }^{41-45}$ and the chemical similarity of metal coordinated nitrogen atoms, ${ }^{31,52}$ it is fairly difficult to discriminate the difference between each other in the $\mathrm{N}$ 1s spectrum. For easy comparison, the $\mathrm{N}$ 1s of pristine CuPc prepared on $\mathrm{Cu}(111)$ with different thickness were also displayed in Fig. S3 in the ESI, $\uparrow$ where it is quite visible that the binding energy of $\mathrm{N} 1 \mathrm{~s}$ in CuPc is nearly same to that in CoPc on $\mathrm{Cu}(111)$. Although no further support could be drawn from $\mathrm{N} 1 \mathrm{~s}$ spectra for the Co substitution process, it is still clear seen that typical $\mathrm{N}$ 1s peaks for the organic macrocycle in Fig. 2 were preserved well during thermal annealing. At this point, it can be concluded that either the original CoPc or the new formed CuPc is kept intact during the whole procedure.

As seen in Fig. 3, the evolution of $\mathrm{C}$ 1s core level spectra is reported for both thin and thick layer of CoPc deposited onto $\mathrm{Cu}$ surface as a function of annealing temperature. Detailed analysis of $\mathrm{C} 1 \mathrm{~s}$ core level spectra first shows that the shape of $\mathrm{C} 1 \mathrm{~s}$ peaks clearly tells the characteristic features for phthalocyanine C 1s spectra. ${ }^{\mathbf{4 0 , 4 8 , 5 3 , 5 4}}$ At monolayer coverage, the $\mathrm{C}$ 1s spectrum is composed by two clearly resolved components with sharp peak, and the intense features are related to the $\mathrm{C}$ atoms within the benzene rings $(284.4 \mathrm{eV})$ and pyrrole $(285.7 \mathrm{eV})$. In the case of 3 ML coverage, there is also a pronounced shoulder attributed to the relative shakeup satellites, which locate at $1.9 \mathrm{eV}$ higher binding energy compared to the main peaks and are well consistent with other reports of metal phthalocyanine..$^{23,44,55}$ Secondly, the peak shape was preserved well for all spectra during annealing either at monolayer or multilayer. Similar to the case of $\mathrm{N} 1 \mathrm{~s}$ spectra, there is a peak shift of $0.4 \mathrm{eV}$ when annealed at $673 \mathrm{~K}$ for monolayer and less than $0.1 \mathrm{eV}$ for multilayer with respect to the spectrum as deposited at RT, which again shall be attributed to the polarization screening effect from metal substrate due to desorption of macrocycle induced by thermal annealing. In the end, as concluded from both $\mathrm{N}$ 1s and $\mathrm{C}$ 1s spectra in Fig. 2 and 3, there is no visible indication for chemical interaction between the benzene ring or pyrrole inside the CoPc macrocycle and the $\mathrm{Cu}(111)$ surface, again supporting the interface interaction mainly localized between the cobalt ion and copper substrate.

In order to reveal the mechanism underlying the experimental observations and get more convincing insights for the atom exchange process occurring at the $\mathrm{CoPc} / \mathrm{Cu}(111)$ interface, we have also performed DFT calculations regarding on the stability of metal phthalocyanine adsorbed on $\mathrm{Cu}(111)$. The binding energy of MPc ( $\mathrm{M}=\mathrm{Cu}$ or $\mathrm{Co})$ on the $\mathrm{Cu}(111)$ surface was calculated utilizing the following formula,

$$
\begin{aligned}
& E_{\text {binding }}(\mathrm{MPc} @ \mathrm{Cu}(111)) \\
& =E(\mathrm{MPc} @ \mathrm{Cu}(111))-E(\mathrm{MPc})-E(\mathrm{Cu}(111))
\end{aligned}
$$

where $E(\mathrm{MPc}), E(\mathrm{Cu}(111))$ and $E(\mathrm{MPc} @ \mathrm{Cu}(111))$ were the total energy of MPc, the $\mathrm{Cu}(111)$ substrate and the complex of MPc/ 
$\mathrm{Cu}(111)$ system, respectively. Results of the calculated binding energies are summarized in Table 1 . As seen, the binding energy was $-0.157 \mathrm{eV}$ for $\mathrm{CuPc} @ \mathrm{Cu}(111)$, and $-0.380 \mathrm{eV}$ for $\mathrm{CoPc} @ \mathrm{Cu}(111)$, where the difference was only $0.223 \mathrm{eV}$, much less than the strength $(\sim 0.5 \mathrm{eV})$ of a hydrogen bonding. ${ }^{56}$ Based on the calculations, a model for the adsorption of MPc on $\mathrm{Cu}(111)$ is shown in Fig. 4, where deformed configurations of both $\mathrm{CoPc}$ and $\mathrm{CuPc}$ can be clearly distinguished upon adsorption on $\mathrm{Cu}(111)$ compared to their planar structures in gas phase, due to the strong molecular substrate interaction. Especially, for CoPc adsorbed on $\mathrm{Cu}(111)$, the cobalt ion is attracted out of the molecular plane towards substrate, while the CuPc molecule is bent mostly on ligands upon adsorption on the $\mathrm{Cu}$ surface. Nevertheless, the stability of CuPc adsorbed on a $\mathrm{Cu}(111)$ surface is similar to that of $\mathrm{CoPc}$ on $\mathrm{Cu}(111)$, which suggests that it is possible to observe the transformation of CoPc into CuPc macrocycle on a copper surface in practice. However, the mechanism underneath is still hidden. Consequently, we further explored the energy of a $\mathrm{Cu}$ atom substituting the Co ion in CoPc to form a CuPc molecule under the following formula,

$$
\begin{aligned}
E_{\text {substituting }} & =[E(\mathrm{CuPc})+E(\mathrm{Co})]-[E(\mathrm{CoPc})+E(\mathrm{Cu}) \\
& =[E(\mathrm{CuPc})-E(\mathrm{Cu})]-[E(\mathrm{CoPc})-E(\mathrm{Co})]
\end{aligned}
$$

where $E(\mathrm{Cu})$ and $E(\mathrm{Co})$ denote the energies of free $\mathrm{Cu}$ and $\mathrm{Co}$ atoms, respectively, $[E(\mathrm{CuPc})-E(\mathrm{Cu})]$ and $[E(\mathrm{CoPc})-E(\mathrm{Co})]$ are the binding energies of $\mathrm{Cu}$ in $\mathrm{CuPc}$ and $\mathrm{Co}$ in CoPc molecule, accordingly. In the end, a substituting energy with $2.031 \mathrm{eV}$ was obtained (Table 1), indicating that a reaction energy of at least $\sim 2 \mathrm{eV}$ is needed to trigger the atom exchange process and form $\mathrm{CuPc}$ macrocycle from original CoPc molecule on the $\mathrm{Cu}(111)$ surface. Nevertheless, such energy can be easily gained by thermal annealing performed in our experiments. However, the appearance of $\mathrm{Co}-\mathrm{Cu}$ atom exchange upon deposition at RT needs to be elaborately investigated, even though only part of CoPc was believed to be substituted, as discussed in Fig. 1. For revealing the origin of atom substation at RT, metal alloying process $^{57}$ has been further taken into consideration in a possible case that a released Co atom from $\mathrm{CoPc}$ replaces a $\mathrm{Cu}$ atom in the $\mathrm{Cu}(111)$ substrate and forms the $\mathrm{Co}-\mathrm{Cu}(111)$ alloy ultimately. Accordingly, alloying energy was calculated based on the following formula,

$$
E_{\text {alloying }}=E(\mathrm{Cu} @ \mathrm{CoCu}(111))-E(\mathrm{Co} @ \mathrm{Cu}(111))
$$

where $E(\mathrm{Co} @ \mathrm{Cu}(111))$ and $E(\mathrm{Cu} @ \mathrm{CoCu}(111))$ are the total energy of a complex with one $\mathrm{Co}$ atom adsorbed on $\mathrm{Cu}(111)$

Table 1 Binding energies of MPc adsorbed on the $\mathrm{Cu}$ (111) surface and

\begin{tabular}{|c|c|c|c|c|c|}
\hline & \multicolumn{2}{|c|}{ MPc@Cu(111) } & \multicolumn{2}{|c|}{$\mathrm{M}$ in MPc } & \multirow{2}{*}{$\begin{array}{l}\text { Co in } \mathrm{CoCu} \\
\text { alloy }\end{array}$} \\
\hline & $\mathrm{CuPc}$ & $\mathrm{CoPc}$ & $\mathrm{Cu}$ & Co & \\
\hline$E_{\text {binding }}(\mathrm{eV})$ & -0.157 & -0.380 & -6.070 & -8.101 & -1.128 \\
\hline$\Delta E(\mathrm{eV})$ & 0.223 & & 2.031 & & \\
\hline
\end{tabular}
the metal atom in MPc ( $M=\mathrm{Cu}$ and $\mathrm{Co})$, as well as the alloying energy of $\mathrm{Co}$ in $\mathrm{CoCu}(111)$

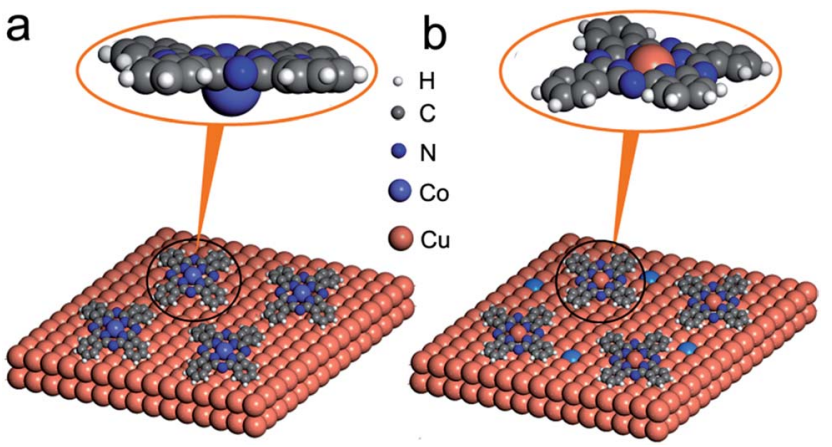

Fig. 4 Configurations of MPc adsorbed on $\mathrm{Cu}(111)$ surface. (a) Deformed structure of CoPc was discovered once adsorbed on the $\mathrm{Cu}(111)$ substrate, where apparently the Co ion was attracted towards substrate from the molecular plane, (b) deformed structure of CuPc on $\mathrm{Cu}(111)$. It is visible that molecular ligands are deformed due to the molecular-substrate interaction.

surface and that with a $\mathrm{Cu}$ atom on $\mathrm{CoCu}(111)$ surface, respectively. The calculated result was $-1.128 \mathrm{eV}$ as shown in Table 1, indicating that the alloying process is exothermal reaction and could therefore provide partial driving force for the transformation from $\mathrm{CoPc} @ \mathrm{Cu}(111)$ to $\mathrm{CuPc} @ \mathrm{Cu}(111)$. In practice, we have also discovered the formation of $\mathrm{Co}-\mathrm{Cu}$ alloy when thin film of cobalt ( $4 \AA$ ) prepared on the $\mathrm{Cu}(111)$ surface was moderately annealed, as present in Fig. S4 in ESI. $\uparrow$ Importantly, one has to bear in mind that the CoPc organic was thermally evaporated onto the $\mathrm{Cu}(111)$ substrate with the sublimation temperature around $700 \mathrm{~K}$, which implies that roughly a thermodynamic energy of about $0.06 \mathrm{eV}\left(E=k_{\mathrm{B}} T\right)$ was applied to the evaporated gas CoPc molecules, although the $\mathrm{Cu}(111)$ substrate was kept at RT. Considering the magnitude order of one degree of freedom with $1 / 2 k_{\mathrm{B}} T(=0.03 \mathrm{eV})$, such embedded thermal vibrations or motions of CoPc macrocycles would provide additional energy to touch off the interface ionsubstitution reaction for some of the adsorbed CoPc molecules on $\mathrm{Cu}(111)$. In a word, the combination of thermal energy supplied by sublimation and an additional energy released by the subsequent formation of $\mathrm{CoCu}$ alloy afterwards, contributes to the ion substitution of $\mathrm{Co}$ in $\mathrm{CoPc}$ on $\mathrm{Cu}(111)$ at RT. Meanwhile, post annealing significantly enhance the ion substation reaction and completely transformed CoPc into CuPc. In another perspective, it can also be understood that the alloying formation of $\mathrm{CoCu}(111)$ will definitely prevent the reversed process of CuPc@CoCu(111) back to CoPc@Cu(111). As a summarize and also for a direct picture, the most possible reaction path occurring at $\mathrm{CoPc} / \mathrm{Cu}(111)$ interface was depicted in Fig. 5, as concluded from DFT calculations.

As reported by Doyle et al., interface atom exchange between nickel porphyrin compounds and $\mathrm{Cu}$ adatoms on a $\mathrm{Cu}(111)$ substrate was undoubtedly demonstrated especially at RT, however, the principle for such substitution has not been clearly addressed. As a consequence, herein, we have consistently explained the experimental observation of cobalt metal state upon adsorption of $\mathrm{CoPc}$ on a $\mathrm{Cu}(111)$ surface, which is originated from the ion substitution of $\mathrm{Co}$ in $\mathrm{CoPc}$ by $\mathrm{Cu}$ adatoms at 


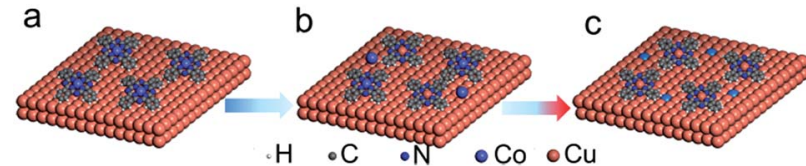

Fig. 5 A possible reaction path occurring at the $\mathrm{CoPc} / \mathrm{Cu}(111)$ interface. (a) As-deposited CoPc on the $\mathrm{Cu}$ (111) substrate, (b) chemical reaction between $\mathrm{Cu}$ atom and $\mathrm{COPC}$ happened at $\mathrm{RT}$ and mild temperature, (c) forming $\mathrm{Cu} / \mathrm{Co}$ alloy in pretty high temperature.

interface. Importantly, the mechanism behind substitution has been properly assigned to the driven force provided either by high-temperature sublimation or post annealing and the following formation of interface alloy, as revealed by detailed DFT calculations.

In short, we have experimentally explored the chemical states of Co 2p, N 1s and $\mathrm{C} 1 \mathrm{~s}$ in with consecution and revealed the interface transmetalation process by DFT calculations, initially induced by the combined contribution from strong molecular-substrate interaction, external thermodynamic energy and interface alloying formation. Spontaneously, one will then easily get curious about the change of valence band structure regarding on new electronic states induced by the interface substitution and molecular-substrate interaction. Besides, a detailed knowledge of the valence band structure is fairly beneficial for the understanding of interfacial electronic structures. Consequently, UPS was recorded as well and analysed elaborately to further exploit the on-surface transmetalation. As plotted in Fig. 6, the valence band of 1 ML CoPc adsorbed on the copper substrate was stacked in sequence as a function of post annealing temperature, as well as the change of work function. While Fig. 6(a) shows the overall change of the valence band structure, a detailed investigation of the valence range from Fermi level $\left(E_{\mathrm{f}}\right)$ to about $1.5 \mathrm{eV}$ below $E_{\mathrm{f}}$ was performed in Fig. 6(b). First, a peak (labeled as A) located at $1.40 \mathrm{eV}$ below $E_{\mathrm{f}}$ is well resolved, which can be attributed to the HOMO of the organic adsorption. Since the HOMO level of CoPc is

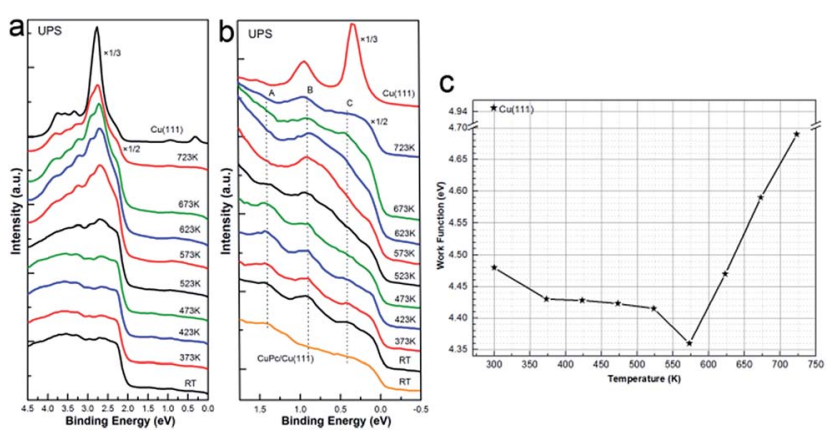

Fig. 6 Valence band structure measured at $h v=21.22 \mathrm{eV}$ for $1 \mathrm{ML}$ of CoPc on $\mathrm{Cu}(111)$ deposited at RT, followed by step-by-step annealing to the indicated temperature. As a guide to eyes, the spectrum of the clean Cu(111) surface was also shown on top. (a) The evolution of UPS as a function of annealing temperature, (b) the zoom-in spectra of panel (a) from Fermi level $\left(E_{f}\right)$ to $1.7 \mathrm{eV}$ below $E_{\mathrm{f}}$. (c) The change of working function (WF) at interface during annealing, the WF of clean $\mathrm{Cu}(111)$ is also indicated as reference. similar to that of CuPc adsorbed on $\mathrm{Cu}(111)$, as shown at the bottom of Fig. 6(b) for direct comparison and consistent with other theoretical reports, ${ }^{52}$ it is implausible to discriminate the contribution from CoPc or CuPc below $573 \mathrm{~K}$ when both of them exist. Notably, peak A gets weaken after further annealing which might be related to desorption of metallized macrocycle, as also discovered by XPS. Moreover, another peak (labelled as B in Fig. 6(b)) located at $0.90 \mathrm{eV}$ below Fermi level is recognized for all the spectra of $\mathrm{CoPc}$ on $\mathrm{Cu}(111)$ at varying annealing temperatures, and this feature is most probably connected to the appearance of Co-Cu compound once Co atom is released from CoPc macrocycle and subsequently interacts with the $\mathrm{Cu}$ substrate, as pointed out by our DFT calculations. Further convincing insight can also be gained from a comparison to the valence band structure of pristine CuPc deposited on the $\mathrm{Cu}(111)$ substrate as shown at the bottom in Fig. 6(b), where no feature located at $0.9 \mathrm{eV}$ below $E_{\mathrm{f}}$ can be resolved, in consistence with the previous report of $\mathrm{CuPc}$ adsorbed on $\mathrm{Cu}$ foil. ${ }^{58}$

As summarized in Fig. 6(c), work function of the clean $\mathrm{Cu}(111)$ is determined to be $4.95 \mathrm{eV}$, while the organic adsorption of CoPc on top dramatically diminish the work function, which is reported to be related with the redistribution of the metal electron cloud or the formation of interface dipole due to the presence of the organic molecules, causing a reduction of the metal work function, ${ }^{\mathbf{5 9 - 6 1}}$ while in our case herein, it can also be related to the appearance of HOMO levels of CoPc on $\mathrm{Cu}(111)$, since the occupied HOMO orbitals will definitely affect the electron redistribution at interface. Interestingly, the decreasing of interface work function is enhanced by the thermal annealing until $573 \mathrm{~K}$, where the transmetalation of CoPc into CuPc gets fully completed, indicating the newly formed $\mathrm{CuPc} / \mathrm{Cu}(111)$ system has a lower work function than the as-prepared $\mathrm{CoPc} / \mathrm{Cu}(111)$ complex. However, the work function starts to recover after $573 \mathrm{~K}$, which is most probably due to the fact that the organic macrocycle begins to desorb with further annealing as confirmed by XPS.

\section{Experimental and calculations}

\section{Photoemission spectroscopy experiments}

All the measurements were performed under ultrahigh vacuum (UHV) conditions with the base pressure better than $5 \times 10^{-10}$ mbar. X-ray photoemission measurements were carried out in the lab using a monochromatic Al-K $\alpha$ radiation $(h \nu=1486.6$ neV), while the ultraviolet photoelectron spectroscopy (UPS) was done with a helium lamp (He I, $h v=21.2 \mathrm{eV})$. Photoelectrons were collected at normal emission to the sample surface with the specs electron analyser (Phoibos 100). The photon energies were calibrated against the $\mathrm{Au} 4 \mathrm{f}_{7 / 2}$ line and the metal Fermi level, respectively. The surface of the $\mathrm{Cu}(111)$ single crystal (Mateck, surface roughness less than $0.01 \mu \mathrm{m}$ with orientation accuracy $<0.1^{\circ}$ ) has been cleaned by repeated cycles of $\mathrm{Ar}^{+}$ sputtering at $1 \mathrm{k}-1.5 \mathrm{k} \mathrm{eV}$, followed by thermal annealing to around $770 \mathrm{~K}$. The surface cleanliness has been checked by XPS afterwards until no carbon or oxygen contamination detected. The CoPc molecule (Sigma-Aldrich) was degassed in vacuum at $600 \mathrm{~K}$ for several hours prior deposition. Thin film of cobalt 
metal was prepared with a home-made e-beam evaporator. Molecules were deposited from a quartz crucible with thermal couple connected onto the substrate kept at room temperature (RT). The deposition rate and film thickness were calculated from the XPS intensity attenuation of $\mathrm{Cu}$ substrate assuming layer-by-layer growth. Herein, one monolayer (ML) is defined as the maximum coverage of molecules adsorbed in direct contact with the substrate as calibrated by annealing a multilayer to 427 $\mathrm{K}$, which is around $4 \AA$ A. The intensity of spectra is normalized to the peak height of the most intense component to highlight the changes in the line shape, which were analyzed afterwards using XPSPEAK.

\section{DFT calculations}

A hexagonal box is used to simulate the unit cell of the adsorption complex, with a dimension of $17.89 \AA \times 17.89 \AA \times$ 19.17 $\AA$ including three layers of $\mathrm{Cu}$ and $15 \AA$ vacuum. The MPc macrocycles are placed into the vacuum and the absorption location of MPc on $\mathrm{Cu}(111)$ surface is determined by the force field simulation method implemented as absorption locator tool in Materials Studio. The MPc@Cu(111) system generated from the force field simulation is used as a input for the accurate total energy calculation.

The total energy calculation is carried out using Projector Augmented Wave (PAW) method ${ }^{62}$ based on the Density Functional Theory $(\mathrm{DFT})^{\mathbf{6 3 , 6 4}}$ as implemented in Vienna $A b$ initio Simulation Package (VASP). ${ }^{65-67}$ The exchange-correlation effect is evaluated by the generalized gradient approximation as parameterized by Perdew, Burke and Ernzerhof (PBE). ${ }^{68}$ $\mathrm{C}(2 \mathrm{~s} 22 \mathrm{p} 2), \mathrm{N}(2 \mathrm{~s} 22 \mathrm{p} 3), \mathrm{Co}(3 \mathrm{~d} 84 \mathrm{~s} 1), \mathrm{Cu}(3 \mathrm{~d} 104 \mathrm{p} 1)$, and $\mathrm{H}(1 \mathrm{~s} 1)$ are treated as valence electrons. A plane wave basis set with a cutoff energy of $600 \mathrm{eV}$ is used. The convergence criterion for electronic self-consistent relaxation is set to $10-5 \mathrm{eV}$. The $\mathrm{Cu}$ atoms in the substrate are fixed while the atoms in the absorbate macrocycles are fully relaxed with a force convergence criteria of $0.01 \mathrm{eV}^{-1}$. The total energies of free standing MPc macrocycles are calculated by placing them into an orthorhombic cell with a size of $17 \times 18 \times 13 \AA^{3}$. Same energy cutoff and convergence criteria with those for total energy calculation of $\mathrm{MPc} @ \mathrm{Cu}(111)$ composite are used.

\section{Conclusions}

Although numerous investigations have been done for transition-metal phthalocyanine adsorbed on metal substrates before, essential details of the electronic structure at interfaces are still unclear, to some extent. In the present work, transmetalation occurring at $\mathrm{CoPc} / \mathrm{Cu}(111)$ interface has been demonstrated by XPS, UPS and well investigated with DFT theory. Whilst the Co(II) oxidation state in CoPc macrocycle is reduced and the $\mathrm{Co}(0)$ state assigned to free cobalt metal atom is increasing with external thermal annealing, as concluded from detailed analysis of Co $2 \mathrm{p}_{3 / 2}$ XP spectra, the substitution of $\mathrm{Co}$ ion in the center of $\mathrm{CoPc}$ by the surface $\mathrm{Cu}$ atom was confirmed. Supported by DFT calculations, strong interaction between CoPc and the copper substrate is proposed; moreover, the mechanism underneath the observed on-surface transmetalation from CoPc to CuPc is revealed, which is jointly contributed by the strong molecular-substrate interaction, external thermodynamic energy and the $\mathrm{Co}-\mathrm{Cu}$ alloying at surface. Furthermore, UPS study also proves the formation of Co-Cu compound at interface following the release of Co from CoPc. While on-surface engineering of metal-organic nanostructures has been an appealing topic to both fundamental research and practical applications nowadays, our report of onsurface transmetalation from $\mathrm{CoPc}$ to $\mathrm{CuPc}$ may predict a controllable approach in solid-state environment to engineer functional metal-organic nanostructures for rich applications in molecular electronics and the related devices.

\section{Acknowledgements}

We thank Dr Yingying Wei and Dr B. V. Tran for fruitful discussions. This work was financially supported by the National Natural Science Foundation of China (91545101 and 11464037), The Hundred Talents Program of Chinese Academy of Sciences, and Shanghai Pujiang Program.

\section{Notes and references}

1 Y. Li, J. Xiao, T. E. Shubina, M. Chen, Z. Shi, M. Schmid, H. Steinrück, J. M. Gottfried and N. Lin, J. Am. Chem. Soc., 2012, 134, 6401-6408.

2 J. P. Collman, R. Boulatov, C. J. Sunderland and L. Fu, Chem. Rev., 2004, 104, 561-588.

3 R. Bonnett, Chem. Soc. Rev., 1995, 24, 19-33.

4 B. R. Takulapalli, G. M. Laws, P. A. Liddell, J. Andréasson, Z. Erno, D. Gust and T. J. Thornton, J. Am. Chem. Soc., 2008, 130, 2226-2233.

5 B. Hulsken, R. Van Hameren, J. W. Gerritsen, T. Khoury, P. Thordarson, M. J. Crossley, A. E. Rowan, R. J. Nolte, J. A. Elemans and S. Speller, Nat. Nanotechnol., 2007, 2, 285-289.

6 F. Sedona, M. Di Marino, D. Forrer, A. Vittadini, M. Casarin, A. Cossaro, L. Floreano, A. Verdini and M. Sambi, Nat. Mater., 2012, 11, 970-977.

7 Y. Zhang and S. N. Riduan, Chem. Soc. Rev., 2012, 41, 20832094.

8 C. Z. Zhang, R. Hao, H. Yin, F. Liu and Y. L. Hou, Nanoscale, 2012, 4, 7326-7329.

9 P. F. Siles, T. Hahn, G. Salvan, M. Knupfer, F. Zhu, D. R. T. Zahn and O. G. Schmidt, Nanoscale, 2016, 8, 86078617.

10 J. V. Barth, G. Costantini and K. Kern, Nature, 2005, 437, 671.

11 J. M. Gottfried, in On-Surface Synthesis, ed. A. Gourdon, Springer, Switzerland, 2016, ch. 7, p. 131.

12 B. W. Caplins, D. E. Suich and A. J. J. Shearer, Phys. Chem. Lett., 2014, 5, 1679-1684.

13 S. Ahmadi, M. N. Shariati, S. Yu and M. Göthelid, J. Chem. Phys., 2012, 137, 084705.

14 H. Peisert, J. Uihlein, F. Petraki and T. Chassé, J. Electron Spectrosc. Relat. Phenom., 2015, 204, 49-60. 
15 A. Zhao, Q. Li, L. Chen, H. Xiang, W. Wang, S. Pan, B. Wang, X. Xiao, J. Yang, J. G. Hou and Q. Zhu, Science, 2005, 309, 1542-1544.

16 S. Stepanow, A. Mugarza, G. Ceballos, P. Moras and J. C. Cezar, Phys. Rev. B: Condens. Matter Mater. Phys., 2010, 82, 014405.

17 J. Schaffert, M. C. Cottin, A. Sonntag, H. Karacuban, C. A. Bobisch, N. Lorente, J. Gauyacq and R. Möller, Nat. Mater., 2013, 12, 223-227.

18 F. Song, J. W. Wells, K. Handrup, Z. S. Li, S. N. Bao, K. Schulte, M. Ahola-Tuomi, L. C. Mayor, J. C. Swarbrick, E. W. Perkins, L. Gammelgaard and P. Hofmann, Nat. Nanotechnol., 2009, 4, 373-376.

19 T. Kroll, V. Y. Aristov, O. V. Molodtsova, Y. A. Ossipyan, D. V. Vyalikh, B. Büchner and M. Knupfer, J. Phys. Chem. A, 2009, 113, 8917-8922.

20 E. Annese, J. Fujii, I. Vobornik and G. Rossi, J. Phys. Chem. C, 2011, 115, 17409-17416.

21 J. Uihlein, M. Polek, M. Glaser, H. Adler, R. Ovsyannikov, M. Bauer, M. Ivanovic, A. B. Preobrajenski, A. V. Generalov, T. Chassé and H. Peisert, J. Phys. Chem. C, 2015, 119, 15240-15247.

22 F. Petraki, H. Peisert, F. Latteyer, U. Aygül, A. Vollmer and T. Chassé, J. Phys. Chem. C, 2011, 115, 21334-21340.

23 F. Petraki, H. Peisert, I. Biswas and T. Chassé, J. Phys. Chem. C, 2010, 114, 17638-17643.

24 K. Diller, A. C. Papageorgiou, F. Klappenberger, F. Allegretti, J. V. Barth and W. Auwärter, Chem. Soc. Rev., 2016, 45, 16291656.

25 P. Hambright and P. B. Chock, J. Am. Chem. Soc., 1974, 96, 3123-3131.

26 C. M. Doyle, J. P. Cunniffe, S. A. Krasnikov, A. B. Preobrajenski, Z. Li, N. N. Sergeeva, M. O. Senge and A. A. Cafolla, Chem. Commun., 2014, 50, 3447-3449.

27 H. Ishii, K. Sugiyama, E. Ito and K. Seki, Adv. Mater., 1999, 11, 605-625.

28 J. Chastian, R. C. King and J. F. Moulder, Handbook of X-ray photoelectron spectroscopy: a reference book of standard spectra for identification and interpretation of XPS data, MN: Physical Electronics, Eden Prairie, 1995.

29 K. Diller, F. Klappenberger, F. Allegretti, A. C. Papageorgiou, S. Fischer, A. Wiengarten, S. Joshi, K. Seufert, D. Écija, W. Auwärter and J. V. Barth, J. Chem. Phys., 2013, 138, 154710.

30 H. Peisert, D. Kolacyak and T. Chassé, J. Phys. Chem. C, 2009, 113, 19244-19250.

31 T. C. Chiang, G. Kaindl and T. Mandel, Phys. Rev. B: Condens. Matter Mater. Phys., 1986, 33, 695.

32 M. Schmid, A. Kaftan, H. Steinrück and J. M. Gottfried, Surf. Sci., 2012, 606, 945-949.

33 J. Uihlein, H. Peisert, M. Glaser, M. Polek, H. Adler, F. Petraki, R. Ovsyannikov, M. Bauer and T. Chassé, J. Chem. Phys., 2013, 138, 081101.

34 J. D. Baran, J. A. Larsson, R. A. J. Woolley, Y. Cong, P. J. Moriarty, A. A. Cafolla, K. Schulte and V. R. Dhanak, Phys. Rev. B: Condens. Matter Mater. Phys., 2010, 81, 075413.
35 M. G. Betti, P. Gargiani, R. Frisenda, R. Biagi, A. Cossaro, A. Verdini, L. Floreano and C. Mariani, J. Phys. Chem. C, 2010, 114, 21638-21644.

36 M. Toader, P. Shukrynau, M. Knupfer, D. R. T. Zahn and M. Hietschold, Langmuir, 2012, 28, 13325; M. Toader, M. Knupfer, D. R. T. Zahn and M. Hietschold, Surf. Sci., 2011, 605, 1510-1515.

37 A. Mugarza, R. Robles, C. Krull, R. Korytár, N. Lorente and P. Gambardella, Phys. Rev. B: Condens. Matter Mater. Phys, 2012, 85, 155437.

38 E. Salomon, P. Amsalem, N. Marom, M. Vondracek, L. Kronik, N. Koch and T. Ango, Phys. Rev. B: Condens. Matter Mater. Phys., 2013, 87, 075407.

39 L. Massimi, M. Angelucci, P. Gargiani, M. G. Betti, S. Montoro and C. Mariani, J. Chem. Phys., 2014, 140, 244704; G. Mette, D. Sutter, Y. Gurdal, S. Schnidrig, B. Probst, M. Iannuzzi, J. Hutter, R. Alberto and J. Osterwalder, Nanoscale, 2016, 8, 7958-7968.

40 Y. Bai, F. Buchner, M. T. Wendahl, I. Kellner, A. Bayer, H. P. Steinruck, H. Marbach and J. M. Gottfried, J. Phys. Chem. C, 2008, 112, 6087-6092; Y. Bai, M. Sekita, M. Schmid, T. Bischof, H.-P. Steinrück and J. M. Gottfried, Phys. Chem. Chem. Phys., 2010, 12, 4336-4344.

41 K. Eguchi, T. Nakagawa, Y. Takagi and T. Yokoyama, J. Phys. Chem. C, 2015, 119, 9805-9811.

42 A. Calabrese, L. Floreano, A. Verdini, C. Mariani and M. G. Betti, Phys. Rev. B: Condens. Matter Mater. Phys, 2009, 79, 115446.

43 M. Abadía, R. González-Moreno, A. Sarasola, G. OteroIrurueta, A. Verdini, L. Floreano, A. Garcia-Lekue and C. Rogero, J. Phys. Chem. C, 2014, 118, 29704-29712.

44 Y. Zhang, S. Wang, A. Demasi, I. Reid, L. F. J. Piper, A. Y. Matsuura, J. E. Downes and K. E. Smith, J. Mater. Chem., 2008, 18, 1792-1978.

45 M. Scardamaglia, C. Struzzi, S. Lizzit, M. Dalmiglio, P. Lacovig, A. Baraldi, C. Mariani and M. G. Betti, Langmuir, 2013, 29, 10440-10447.

46 S. Yu, S. Ahmadi, C. Sun, P. T. Z. Adibi, W. Chow, A. Pietzsch and M. M. Göthelid, J. Chem. Phys, 2012, 136, 154703.

47 T. Lukasczyk, K. Flechtner, L. R. Merte, N. Jux, F. Maier, J. M. Gottfried and H. Steinrück, J. Phys. Chem. C., 2007, 111, 3090-3098.

48 Y. Bai, F. Buchner, I. Kellner, M. Schmid, F. Vollnhals, H. Steinrueck, H. Marbach and J. M. Gottfried, New J. Phys., 2009, 11, 125004.

49 R. Liu, J. Duay, T. Lane and S. Bok Lee, Phys. Chem. Chem. Phys., 2010, 12, 4309-4316.

50 K. Diller, F. Klappenberger, M. Marschall, K. Hermann, A. Nefedov, C. Wöll and J. V. Barth, J. Chem. Phys., 2012, 136, 014705.

51 G. Di Santo, C. Castellarin-Cudia, M. Fanetti, B. Taleatu, P. Borghetti, L. Sangaletti, L. Floreano, E. Magnano, F. Bondino and A. Goldoni, J. Phys. Chem. C, 2011, 115, 4155-4162.

52 F. Klappenberger, A. Weber-Bargioni, W. Auwärter, M. Marschall, A. Schiffrin and J. V. Barth, J. Chem. Phys., 2008, 129, 214702. 
53 H. Peisert, M. Knupfer and J. Fink, Surf. Sci., 2002, 515, 491498.

54 A. Ruocco, F. Evangelista, R. Gotter, A. Attili and G. Stefani, J. Phys. Chem. C, 2008, 112, 2016-2025.

55 S. Ahmadi, B. Agnarsson, I. Bidermane, B. M. Wojek, Q. Noel, C. Sun and M. Gothelid, J. Chem. Phys., 2014, 140, 174702 .

56 S. J. Grabowski, J. Phys. Org. Chem., 2004, 47, 18-31.

57 T. Allmers and M. Donath, Surf. Sci., 2011, 605, 1875-1924.

58 S. Sinha and M. Mukherjee, Appl. Surf. Sci., 2015, 353, 540547.

59 H. Peisert, M. Knupfer and J. Fink, Appl. Phys. Lett., 2002, 81, 2400.

60 X. Crispin, V. Geskin, A. Crispin, J. Cornil, R. Lazzaroni, W. R. Salaneck and J. Brédas, J. Am. Chem. Soc., 2002, 124, 8131-8141.
61 P. S. Bagus, V. Staemmler and C. Woll, Phys. Rev. Lett., 2002, 89, 096104.

62 P. E. Blöchl, Phys. Rev. B: Condens. Matter Mater. Phys., 1994, 50, 17953.

63 P. Hohenberg and W. Kohn, Phys. Rev., 1964, 136, B864.

64 W. Kohn and L. J. Sham, Phys. Rev., 1965, 140, A1133.

65 G. Kresse and J. Hafner, Phys. Rev. B: Condens. Matter Mater. Phys., 1993, 47, 558; G. Kresse and J. Hafner, Phys. Rev. B: Condens. Matter Mater. Phys., 1994, 49, 14251.

66 G. Kresse and J. Furthmüller, Phys. Rev. B: Condens. Matter Mater. Phys., 1996, 54, 11169.

67 G. Kresse and D. Joubert, Phys. Rev. B: Condens. Matter Mater. Phys., 1999, 59, 1758.

68 J. P. Perdew, K. Burke and M. Ernzerhof, Phys. Rev. Lett., 1996, 77, 3865. 